Pacific Journal of Mathematics

GENERATORS OF THE MAXIMAL IDEALS OF $A(\bar{D})$ 


\title{
GENERATORS OF THE MAXIMAL IDEALS OF $A(\bar{D})$
}

\author{
NILS ØVRELID
}

\begin{abstract}
Let $A=A(\bar{D})$ be the sup norm algebra of functions continuous in $\bar{D}$ and holomorphic in $D$, where $D$ is a bounded, strictly pseudoconvex domain in $\mathrm{C}^{n}$. This paper gives necessary and sufficient local conditions that a subfamily of $A$ generates the maximal ideal $\mathscr{M}_{w}(\bar{D})$ of functions in $A$ vanishing at $w \in \bar{D}$. In particular, it shows that $\mathscr{K}_{w}(\bar{D})$ is generated by $z_{1}-w_{1}, \cdots, z_{n}-w_{n}$ when $W \in D$.
\end{abstract}

In [3], Gleason shows that if $m$ is an (algebraically) finitely generated maximal ideal of a commutative Banach algebra $A$, the maximal ideal space $\mathscr{L}_{A}$ can be given an analytic structure near $m$, in terms of which the Gelfand transforms of the elements of $A$ are holomorphic functions.

In a sense, the results of this paper go in the opposite direction. We consider a bounded domain $D$ in $C^{n}$, with $C^{2}$ strictly pseudoconvex boundary, and study the algebra $A=A(\bar{D})$ of functions continuous on $\bar{D}$ and holomorphic in $D$. By a recent result, Henkin [4], Kerzman [7], Lieb [9], $A$ equals the closure in $C(\bar{D})$ of the algebra $O(\bar{D})$ of functions holomorphic in some neighbourhood of $\bar{D}$, from which it follows that $\mathscr{A l}_{A} \approx \bar{D}$.

We first fix the notation. If $w \in \bar{D}$, $\mathscr{C}_{w}$ denotes the maximal ideal of the ring $O_{w}$ of germs of holomorphic functions at $w$, while $\mathscr{C}_{w}(\bar{D})$ is the maximal ideal in $A$ of functions vanishing at $w$. If $f$ is a function on some neighbourhood of $w, f_{w}$ denotes the germ of $f$ at $w$.

Theorem 1. Let $w \in D$, and $f_{1}, \cdots, f_{N} \in A$. Then $f_{1}, \cdots, f_{N}$ generate $\mathscr{C}_{w}(\bar{D})$ if and only if

(1) $f_{1_{w}}, \cdots, f_{N_{w}}$ generate $\mathscr{C}_{w}$, and

(2) $w$ is the only common zero of $f_{1}, \cdots, f_{N}$ in $\bar{D}$.

Corollary. If $w \in D, z_{1}-w_{1}, \cdots, z_{n}-w_{n}$ generate $\mathscr{C}_{w}(\bar{D})$.

Below we give the more general theorem 2, which also gives a similar characterization of generators of $\mathscr{C}_{w}(\bar{D})$ when $w \in \partial D$. When $n=2$, Kerzman and Nagel [8] have shown that $z_{1}-w_{1}$ and $z_{2}-w_{2}$ generate $\mathscr{C}_{w}(\bar{D})$ when $w \in D$, as well as similar results for algebras with Hölder norms. I want to thank Dr. Kerzman for sending me a copy of his thesis [7], where these results are stated.

The main tool in the proof is the following result, which is proved in [11]: 
Lemma 1. Suppose $u \in C_{(0, q)}^{\infty}(D)$ is bounded, with $\bar{\partial} u=0, q \geqq 1$. Then there exists $a v \in C_{(0, q-1)}^{\infty}(D)$ with $\bar{\partial} v=u$, such that $v$ has a continuous extension to $\bar{D}$.

A closely related result is given in Lieb [10], while a stronger result for $(0,1)$-forms, involving Hölder estimates, is given in Kerzman [7].

It is convenient to prove first a more general result. If $U$ is open in $\bar{D}$, let $H(U)$ denote functions in $C(U)$ that are holomorphic in $D \cap U$. When $w \in \bar{D}$, we define $H_{w}=\underset{U \ni w}{\lim } H(U)$, so $H_{w}$ is the space of germs at $w$ of continuous functions on $\bar{D}$ that are holomorphic in $D$. It is easy to see that $H$ is the sheaf of $A$-holomorphic functions in the sense of [2].

Proposition 1. Let $D$ be as above, $w \in \bar{D}$, and suppose $f_{1}, \cdots, f_{N}$ have $w$ as their only common zero. We let I denote the ideal in $A$ generated by $f_{1}, \cdots, f_{N}$, and $I_{w}$ the ideal in $H_{w}$ generated by $f_{1 w}, \cdots$, $f_{N w}$. If $f \in A$ and $f_{w} \in I_{w}$, then $f \in I$.

Proof. By assumption, we may write $f=\sum_{i=1}^{N} g_{i} \cdot f_{i}$ on a neighbourhood $U$ of $w$ in $\bar{D}$, with $g_{1}, \cdots, g_{N} \in H(U)$. We want to write $f=\sum_{i=1}^{N} h_{i} \cdot f_{i}$, with $h_{1}, \cdots, h_{N} \in A$, and shall first solve the problem differentiably. As the sets $N_{i}=\left\{z \in \bar{D} \backslash\{w\}: f_{i}(z)=0\right\}, i=1, \cdots, N$, are closed in $C^{n} \backslash\{w\}$, it is well known how to construct $\widetilde{\varphi}_{1}, \cdots, \widetilde{\varphi}_{N}$ with $\widetilde{\varphi}_{i}=0$ on a neighbourhood of $N_{i}, i=1, \cdots, N$, that form a $C^{\infty}$ partition of unity on $C^{n} \backslash\{w\}$. Choose $\varphi_{0} \in C_{0}^{\infty}\left(U^{\prime}\right)$, where $U^{\prime} \cap \bar{D}=U$, with $\varphi_{0}=1$ on a neighbourhood $U_{1}$ of $\mathrm{w}$, and define $\varphi_{i}=\left(1-\varphi_{0}\right) \cdot \widetilde{\varphi}_{i}, i=$ $1, \cdots, N$.

If we define

$$
g_{i}^{\prime}=\varphi_{0} \cdot g_{i}+\frac{\varphi_{i} \cdot f}{f_{i}} \text {, clearly } \sum_{i=1}^{N} g_{i}^{\prime} \cdot f_{i}=f \text { on } \bar{D} .
$$

The $g_{i}^{\prime} s \in C^{\infty}(D) \cap C(\bar{D})$, and are holomorphic in $U_{1} \cap D$.

We want to use Lemma 1 to modify the $g_{i}^{\prime} s$ to get $h_{i}$ 's in $A$. To handle the combinatorial difficulties, we apply the homological argument of [6].

Notation. $L_{r}=\left\{u \in C_{(0, r)}^{\infty}(D), u\right.$ and $\bar{\partial} u$ have bounded coefficients $\}$, while $L_{r}^{s}=L_{r} \otimes_{c} \Lambda^{s} C^{N}, 0 \leqq r, s$.

If we choose a basis $e_{1}, \cdots, e_{N}$ in $C^{N}$, the elements in $L_{r}^{s}$ may be written uniquely as $\sum_{|I|=s} u_{I} \otimes e^{I}$, where $u_{I} \in L_{r}, e^{I}=e_{i_{1}} \wedge \cdots \wedge e_{i_{s}}$, and we sum over strictly increasing sequences $I=\left(i_{1}, \cdots, i_{s}\right)$. We define $\bar{\partial}$ on $L_{r}^{s}$ by $\bar{\partial}(u \otimes \omega)=(\bar{\partial} u) \otimes \omega$ and linearity. Clearly 
$\bar{\partial} L_{r}^{s} \subset L_{r+1}^{s}$, and lemma 1 gives:

LEMMA $1^{\prime}$. If $k \in L_{r}^{s}$ and $\bar{\partial} k=0, r \geqq 1$, there exists a $k^{\prime} \in L_{r-1}^{s}$, such that $\bar{\partial} k^{\prime}=k$, and $k^{\prime}$ has a continuous extension to $\bar{D}$.

The product determined by $(u \otimes \omega) \cdot\left(u^{\prime} \otimes \omega^{\prime}\right)=\left(u \wedge u^{\prime}\right) \otimes\left(\omega \wedge \omega^{\prime}\right)$ is clearly a bilinear map $L_{r}^{s} \times L_{r \prime}^{s \prime} \rightarrow L_{r+r}^{s+s \prime}$.

Let $e_{1}^{*}, \cdots, e_{N}^{*}$ be the reciprocal basis to $e_{1}, \cdots, e_{N}$, so $\left\langle e_{i}^{*}, e_{j}\right\rangle=$ $\delta_{i j}$. We define $P_{f}: L_{r}^{s} \rightarrow L_{r}^{s-1}$ by

$$
\left.P_{f}(d \otimes \omega)=\sum_{i=1}^{N}\left(f_{i} \cdot u\right) \otimes\left(e_{i}^{*}\right\rfloor \omega\right), \text { and linearity. }
$$

(For the definition of $\rfloor$, se [12] Ch. 1.) $P_{f}: L_{r}^{1} \rightarrow L_{r}^{0}$ maps $\sum_{i=1}^{N} u_{i} \otimes e_{i}$ to $\sum_{i=1}^{N} f_{i} \cdot u_{i}$; in particular, $P_{f} g^{\prime}=f$, when $g^{\prime}=\sum_{i=1}^{N} g_{i}^{\prime} \otimes u_{i}$.

A simple computation gives $P_{f}^{2}=0$, while the derivation property of $」$ gives

$$
P_{f}\left(k \cdot k^{\prime}\right)=\left(P_{f} k\right) \cdot k^{\prime}+(-1)^{s} k \cdot P_{f} k^{\prime}
$$

when $k \in L_{r}^{s}$.

Let $M_{r}^{s}=\left\{k \in L_{r}^{s}:\left.k\right|_{U_{1}}=0\right\}$.

Lemma 2. The complex $0 \leftarrow M_{r}^{0} \stackrel{P_{f}}{\rightarrow} M_{r}^{1} \stackrel{P_{f}}{\rightarrow} \cdots \stackrel{P_{f}}{\rightarrow} M_{r}^{v} \leftarrow 0$ is exact.

Proof. Let $\varphi \in C^{\infty}\left(\boldsymbol{C}^{N}\right)$ be zero near $w$ and one outside $U_{1}$. We put $k_{0}=\sum_{i=1}^{N}\left(\varphi \cdot \widetilde{\varphi}_{i}\right) / f_{i} \otimes e_{i}$. Clearly $k_{0} \in L_{0}^{1}$, and $P_{f} k_{0} \in L_{0}^{0}$ is identically one in $D \backslash U_{1}$. If $k \in M_{r}^{s}$ and $P_{f} k=0, k_{0} \cdot k \in M_{r}^{s+1}$, and by (i), $P_{f}\left(k_{0} \cdot k\right)=\left(P_{f} k_{0}\right) \cdot k=k$.

As $f_{1}, \cdots, f_{N}$ are holomorphic in $D, P_{f}$ and $\bar{\partial}$ commute.

Lemma 3. If $k \in M_{r}^{s}$ and $P_{f} k=\bar{\partial} k=0$, there exists $a k^{\prime} \in L_{r}^{s+1}$, with $P_{f} k^{\prime}=k$ and $\bar{\partial} k^{\prime}=0$.

This is trivially true when $r>n$, and the proof goes by downward induction on $r$. Suppose the lemma is valid for $r+1$. By Lemma 2 , there exists a $k_{1} \in M_{r}^{s+1}$ with $P_{f} k_{1}=k$. Clearly $\bar{\partial} M_{r}^{s+1} \subset M_{r+1}^{s+1}$, while $P_{f} \bar{\partial} k_{1}=\bar{\partial} P_{f} k_{1}=0$. Using the induction hypothesis, we can find $k_{2} \in L_{r+1}^{s+2}$ with $P_{f} k_{2}=\bar{\partial} k_{1}$ and $\bar{\partial} k_{2}=0$. By Lemma $1^{\prime}, k_{2}=\bar{\partial} k_{3}$, with $k_{3}$ $\in L_{r}^{s+2}$. If we put $k^{\prime}=k_{1}-P_{f} k_{3}$, we get $k^{\prime} \in L_{r}^{s+1}$, with $\bar{\partial} k^{\prime}=\bar{\partial} k_{1}-$ $P_{f} \bar{\partial} k_{3}=0$, and $P_{f} k^{\prime}=P_{f} k_{1}-P_{f}^{2} k_{3}=k$. This completes the induction step. 
Proof of Proposition 1. As the $g_{i}^{\prime}$ s are holomorphic in $U_{1} \cap D$, $\bar{\partial} g^{\prime} \in M_{1}^{1}$. Applying Lemma $1^{\prime}$ and Lemma 3 , we find a $k \in L_{0}^{2}$, with $\bar{\partial} P_{f} k=P_{f} \bar{\partial} k=\bar{\partial} g^{\prime}$, such that $k$ is continuous on $\bar{D}$. If $h=g^{\prime}-P_{f} k$, $\bar{\partial} h=0$. Writing $h=\sum_{i=1}^{N} h_{i} \otimes \mathrm{e}_{i}$, this means that $h_{1}, \cdots, h_{N} \in A$, and $\sum_{i=1}^{N} h_{i} \cdot f_{i}=f$.

THEOREM 2. Let $w \in \bar{D}$, and let $M_{w}$ denote the unique maximal ideal of $H_{w}$. The family $\left(f_{i}\right)_{i \in I}$ in $A$ generates $\mathscr{L}_{w}(\bar{D})$ if and only if

(1) $\left(f_{i_{w}}\right)_{i \in I}$ generates $M_{w}$, and

(2) $w$ is the only common zero of functions $f_{i}$ in $\bar{D}$

Proof. I. The sufficiency of (1) and (2): If $f \in \mathscr{C}_{w}(\bar{D})$, we have $f_{w} \in M_{w}$, and by (1) $f_{w}$ belongs to some ideal $\left[f_{i_{1}, w}, \cdots, f_{i_{M, w}}\right]$. As $\left(z_{1}-w_{1}\right)_{w}, \cdots, f\left(z_{n}-w_{n}\right)_{w}$ belong to $M_{w}$, the functions $z_{i}-w_{i} ; i=$ $1, \cdots, n$, may be expressed as linear combinations of functions $f_{i_{M+1}}$, $\cdots, f_{i_{P}}$ in the family on some open neighbourhood $V$ of $w$ in $\bar{D}$. Then $f_{i_{M+1}}, \cdots, f_{i_{P}}$ have $w$ as their only common zero in $V$. By condition (2) and the compactness of $\bar{D} \backslash V$, there exist $f_{i_{P+1}}, \cdots, f_{i_{N}}$ in the family with no common zeroes outside $V$. Now proposition 1 implies that $f$ $\in\left[f_{i_{1}}, \cdots, f_{i_{N}}\right]$.

II. The necessity of (1) and (2): If $\left(f_{i}\right)_{i \in I}$ generate $\mathscr{L}_{w}(\bar{D})$, condition (2) follows from the fact that $A$ separates points in $\bar{D}$. Condition (1) follows from

Proposition 2. The germs at $w$ of elements in $\mathscr{C l}_{w}(\bar{D})$ generate $M_{w}$.

The following proof of Proposition 2 was kindly communicated to me by Dr. R. M. Range, and replaces a more complicated argument of my own:

When $w \in D, z_{1}-w_{1}, \cdots, z_{n}-w_{n}$ generate $\mathscr{l}_{w}=M_{w}$. Thus we may assume $w \in \partial D$, and consider an $f \in H(U \cap \bar{D})$ with $f(w)=0$, where $U$ is some neighbourhood of $w$ in $C^{n}$. We choose $\varphi \in C_{0}^{\infty}(U)$ such that $\varphi \equiv 1$ on a smaller neighbourhood $V$ of $w$. As $D$ is strictly pseudoconvex, we may extend it inside $V$ to a strictly pseudoconvex domain $D^{\prime}$ containing $w$. As $\bar{\partial}(\varphi \cdot f)$ vanishes on $V \cap D$, it may be extended by zero to a smooth, bounded, $\bar{\partial}$-closed $(0,1)$-form $\omega$ on $D^{\prime}$. By Lemma 1, the equation $\bar{\partial} g=\omega$ has a solution in $C^{\infty}\left(D^{\prime}\right) \cap C\left(\bar{D}^{\prime}\right)$, and we may assume $g(w)=0$. As $g$ is holomorphic in $D^{\prime} \cap V$, we may write it near $w$ as $g=\sum_{i=1}^{n} g_{i}\left(z_{i}-w_{i}\right)$, with $g_{1}, \cdots, g_{n}$ holomorphic. Thus $f_{w}=(\varphi \cdot f-g)_{w}+\sum_{i=1}^{n} g_{i_{w}}\left(z_{i}-w_{i}\right)_{w}$, and $\varphi \cdot f-\left.g\right|_{\bar{D}} \epsilon$ $\mathscr{C}_{w}(\bar{D})$. 
When $w \in D$ and $I$ is finite, Theorem 2 reduces to theorem 1 . If $w \in \partial D$, it follows from Gleason's result that $\mathscr{K}_{w}(\bar{D})$ is not finitely generated. If $M_{w}$ were finitely generated, it would by Proposition 2 be generated by finitely many elements of $A$, which implies by the argument of $I$ that $\mathscr{A}_{w}(\bar{D})$ must be finitely generated. Thus $M_{w}$ is not finitely generated when $w \in \partial D$. (This may also be proved in a more direct fashion).

Note. The Corollary to Theorem 1 has also been proved by G. M. Henkin in Bull. Acad. Polon. Sci., 24 (1971) 37-42, and by I. Lieb in Math. Ann., 190 (1970-71) 6-44, which contains a detailed version of [10].

\section{REFERENCES}

1. J. Dieudonné, Foundations of Modern Analysis, Academic Press 1960.

2. T. W. Gamelin, Uniform Algebras, Prentice Hall 1969.

3. A. Gleason, Finitely generated ideals in Banach algebras, J. Math. Mech., 13 (1964), 125-32.

4. G. M. Henkin, Integral representations of functions holomorphic in strictly pseudoconvex domains and some applications, Mat. Sbornik, 78 (1969), 611-32. (Math. USSR Sbornik Vol. 7 (1969).)

5. L. Hörmander, Introduction to Complex Analysis in Several Variables, van Nostrand 1966.

6. 73 (1967), 943-49. , Generators of some rings of analytic functions, Bull. Amer. Math. Soc.,

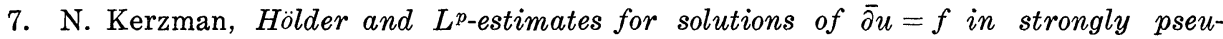
doconvex domains, Comm. Pure. Appl. Math. 24 (1971) 301-80.

8. N. Kerzman and A. Nagel, Finitely generated ideals in certain function algebras Journal of Functional Analysis. 7 (1971) 212-15.

9. I. Lieb, Ein approximationssatz auf streng pseudoconvexen gebieten, Math. Ann., 184 (1969), 56-60.

10. , Beschränktkeitsaussagen für den $d^{\prime \prime}$-operator, Nachr. Ak. Wiss. Gött. II. Math. Phys. Kl. 1970, 1, 7 p.

11. N. Øvrelid, Integral representation formulas and $L^{p}$-estimates for the $\bar{\partial}$-equation, (to appear in Math. Scand.).

12. S. Sternberg, Lectures on Differential Geometry, Prentice Hall 1964.

Received August 6, 1970. Dr. Range's proof received June 28, 1971.

UNIVERSITETET I OSLO 



\section{PACIFIC JOURNAL OF MATHEMATICS}

\section{EDITORS}

H. SAMELSON

Stanford University

Stanford, California 94305

C. R. HobBY

University of Washington

Seattle, Washington 98105
J. DugundjI

Department of Mathematics

University of Southern California

Los Angeles, California 90007

RICHARD ARENS

University of California

Los Angeles, California 90024

\section{ASSOCIATE EDITORS}

E. F. BECKENBACH

B. H. NeUMaNN

F. WOLF

K. YoshidA

\section{SUPPORTING INSTITUTIONS}

UNIVERSITY OF BRITISH COLUMBIA

CALIFORNIA INSTITUTE OF TECHNOLOGY

UNIVERSITY OF CALIFORNIA

MONTANA STATE UNIVERSITY

UNIVERSITY OF NEVADA

NEW MEXICO STATE UNIVERSITY

OREGON STATE UNIVERSITY

UNIVERSITY OF OREGON

OSAKA UNIVERSITY
UNIVERSITY OF SOUTHERN CALIFORNIA STANFORD UNIVERSITY

UNIVERSITY OF TOKYO

UNIVERSITY OF UTAH

WASHINGTON STATE UNIVERSITY UNIVERSITY OF WASHINGTON

$\stackrel{*}{*} \stackrel{*}{*} \stackrel{*}{*}$ AMERICAN MATHEMATICAL SOCIETY
NAVAL WEAPONS CENTER 


\section{Pacific Journal of Mathematics}

Vol. 39 , No. 1

May, 1971

Charles A. Akemann, A Gelfand representation theory for $C^{*}$-algebras ....

Sorrell Berman, Spectral theory for a first-order symmetric system of

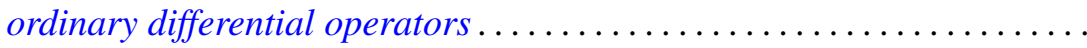

Robert L. Bernhardt, III, On splitting in hereditary torsion theories ........

J. L. Brenner, Geršgorin theorems, regularity theorems, and bounds for determinants of partitioned matrices. II. Some determinantal identities ..........................................

Robert Morgan Brooks, On representing $F^{*}$-algebras .............. 51

Lawrence Gerald Brown, Extensions of topological groups........... 71

Arnold Barry Calica, Reversible homeomorphisms of the real line ........ 79

J. T. Chambers and Shinnosuke Oharu, Semi-groups of local Lipschitzians in

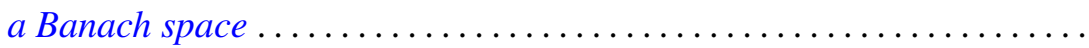

Thomas J. Cheatham, Finite dimensional torsion free rings .............

Byron C. Drachman and David Paul Kraines, A duality between

transpotence elements and Massey products ...................

Richard D. Duncan, Integral representation of excessive functions of a

Markov process ......................................

George A. Elliott, An extension of some results of Takesaki in the reduction

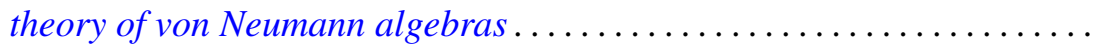

Peter C. Fishburn and Joel Spencer, Directed graphs as unions of partial

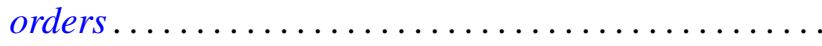

Howard Edwin Gorman, Zero divisors in differential rings ...

Maurice Heins, A note on the Löwner differential equations...

Louis Melvin Herman, Semi-orthogonality in Rickart rings. .

David Jacobson and Kenneth S. Williams, On the solution of linear G.C.D.

equations

Michael Joseph Kallaher, On rank 3 projective planes ... . .

Donald Paul Minassian, On solvable $O^{*}$-groups ...........

Nils Øvrelid, Generators of the maximal ideals of $A(\bar{D})$

Mohan S. Putcha and Julian Weissglass, A semilattice decomposition into

semigroups having at most one idempotent ............

Robert Raphael, Rings of quotients and $\pi$-regularity ....

J. A. Siddiqi, Infinite matrices summing every almost periodic sequence. .

Raymond Earl Smithson, Uniform convergence for multifunctions ...

Thomas Paul Whaley, Mulitplicity type and congruence relations in

universal algebras...

Roger Allen Wiegand, Globalization theorems for locally finitely generated modules... 